\title{
Sails: automated model building of carbohydrates
}

\author{
M. Atanasova, J. Agirre

 \\ jon.agirre@york.ac.uk
}

Carbohydrates are central to many biological processes. As protein glycosylation, they mediate interactions in recognition processes in cancer, viral infection, fertilisation. For example, the surfaces of viruses and antibodies are often covered in carbohydrates, which has been exploited in the development of vaccines and therapies. To further such efforts, it is important to have a good understanding of their 3D structure.

A software, Sails (Software for Automated Identification of Linked Sugars), has been developed to build sugars into electron density/potential maps automatically. It currently works for N-glycosylation and ligands, with plans to expand it for O-glycosylation. Sails uses a database of sugar fingerprints. These fingerprints are generated by superposing sugars from high-resolution structures in the PDB in their minimal-energy conformation, with correct anomeric forms and stereochemistry. The resulting fingerprints contain a set of coordinates of the atoms, plus a set of peaks and voids. Peaks represent map places where the electron density/potential of the fingerprint is expected to be high, while voids are places where map density values are likely to be close to zero. Figure 1 represents examples of sugars detected with Sails. As part of this presentation, I will discuss the fingerprinting method, its application to sugars nd real world results from using Sails on published datasets.

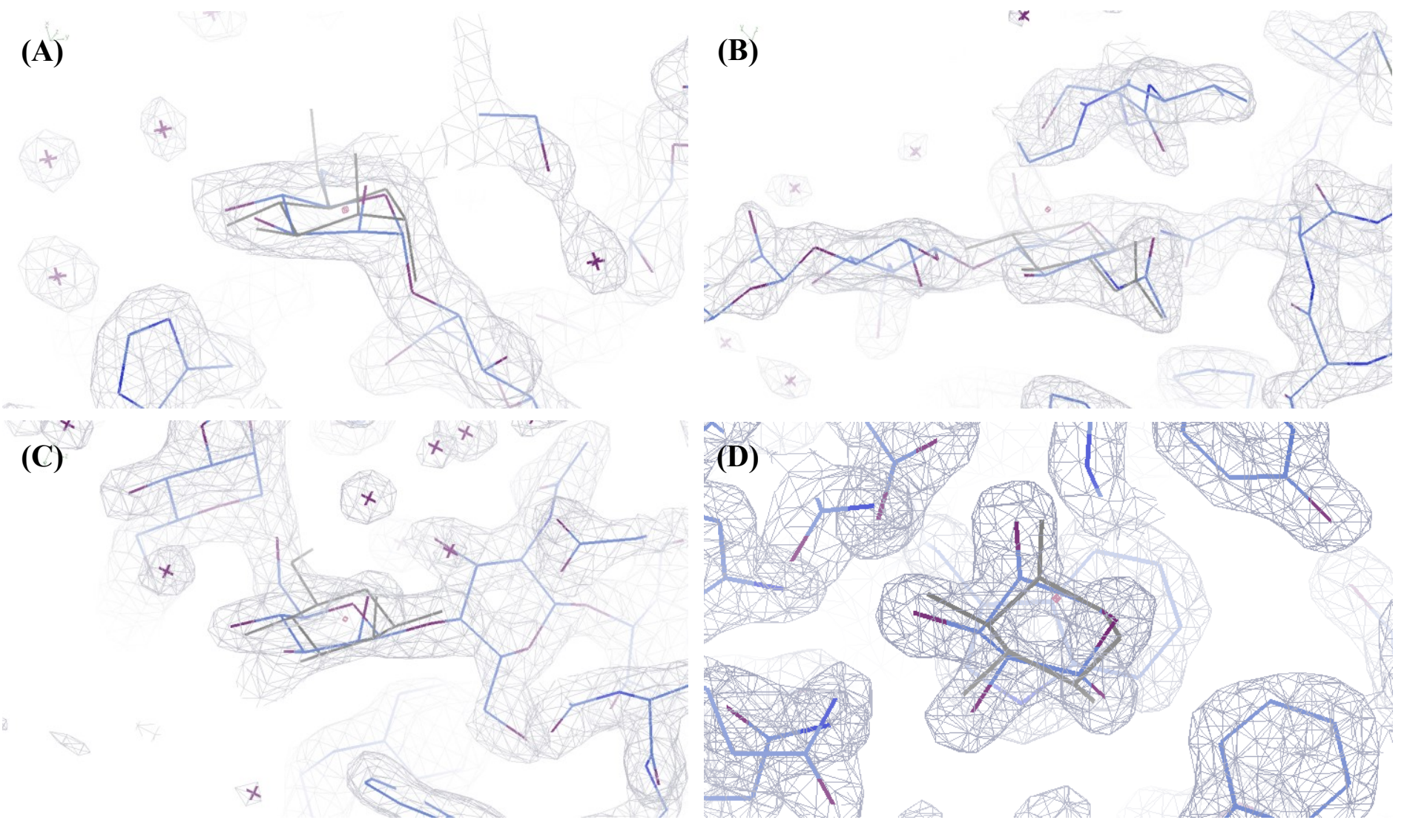

Figure 1. Sugar detected by Sails. The detected sugar is shown in grey and the deposited model is shown in blue. (A) alpha-Dmannopyranose (B) N-acetyl-beta-D-glucosamine (C) beta-D-mannopyranose (D) alpha-L-arabinopyranose. 